\title{
Protocol of a prospective cohort study of the effect of different methods of drainage of neuropathic bladder on occurrence of symptomatic urinary infection, and adverse events related to the urinary drainage system in spinal cord injury patients Subramanian Vaidyanathan*1, Bhakul M Soni ${ }^{1}$, Singh Gurpreet ${ }^{1}$,

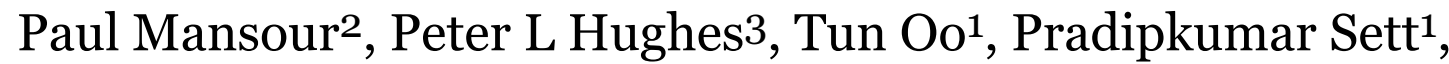 Keith F Parsons4 and John C Davies5
}

\begin{abstract}
Address: ${ }^{1}$ Regional Spinal Injuries Centre, District General Hospital, Southport PR8 6PN, UK, ${ }^{2}$ Department of Cellular Pathology, District General Hospital, Southport PR8 6PN, UK, 3Department of Radiology, District General Hospital, Southport PR8 6PN, UK, 4Department of Urology, Royal Liverpool University Hospital, Liverpool L7 8XP, UK and 5Computing Services Department, The University of Liverpool, Liverpool L69 3BX, UK

E-mail: Subramanian Vaidyanathan* - vaidyanathansiu@hotmail.com; Bhakul M Soni - Bhakul.Soni@mail.soh-tr-nwest.nhs.uk; Singh Gurpreet - Gurpreet.Singh@mail.soh-tr.nwest.nhs.uk; Paul Mansour - Paul.Mansour@mail.soh-tr.nwest.nhs.uk; Peter L Hughes - Peter.Hughes@mail.soh-tr.nwest.nhs.uk; Tun Oo - drtunoo@hotmail.com; Pradipkumar Sett - Pradip.Sett@mail.sohtr.nwest.nhs.uk; Keith F Parsons - Keith_Parsons@hotmail.com; John C Davies - J.C.Davies@liverpool.ac.uk

*Corresponding author
\end{abstract}

This article is available from: http://www.biomedcentral.com/I47I-2490/I/2

(C) 200 I Vaidyanathan et al; licensee BioMed Central Ltd. Verbatim copying and redistribution of this article are permitted in any medium for any noncommercial purpose, provided this notice is preserved along with the article's original URL. For commercial use, contact info@biomedcentral.com

\begin{abstract}
Background: To present a protocol of a prospective, cohort study in which four groups of spinal cord injury (SCl) patients will participate. (Patients with indwelling urethral catheter; patients who perform intermittent catheterisation without wearing a penile sheath; patients who perform intermittent catheterisation and wear penile sheath as well; and patients with penile sheath drainage).

Objectives: (I) What is the incidence of symptomatic urinary infection in men with spinal cord injury who use different types of bladder drainage? (2) Which are predisposing factors for the occurrence of symptomatic urinary infection in men with spinal cord injury who practise different methods of bladder drainage? (3) What is the incidence of catheter and urinary drainage system-related adverse events in the four groups of $\mathrm{SCl}$ patients?

Patients: The criteria for inclusion are as follow: (I) Male patients with neuropathic bladder due to spinal cord injury, who are registered with the Regional Spinal Injuries Centre, Southport, England. (2) Age: 18 years or above. (3) Patients who are willing to give informed consent for participation in the study. (4) Patients willing to be contacted every two weeks by a staff of the spinal unit for 36 months. (5) Patients who are willing to maintain an accurate record of adverse events related to urinary catheter and urinary drainage system and predisposing factors for the occurrence of symptomatic urinary infection. (6) Patients, who are stabilised in a particular method of bladder drainage, and therefore, unlikely to make a permanent change in the method of bladder drainage (e.g. from penile sheath drainage to the use of long-term indwelling catheter) during a foreseeable future.

Methods: The participants will be observed for a period of 36 months. A staff of the spinal injuries unit will contact the participants by telephone every two weeks on a mutually agreed day and time. The information obtained during this standardised telephonic interview conducted once in two weeks will be entered in a database. When a participant develops symptom(s) suggestive of urinary infection, he will undergo urine and blood tests, and imaging studies of the urinary tract.

Conclusion: This study will provide information regarding the occurrence of symptomatic urinary infection, predisposing factors for development of urinary infection, and adverse events related to urinary catheter and urinary drainage system in $\mathrm{SCl}$ patients using different methods of bladder drainage.
\end{abstract}




\section{Introduction}

Urinary infection is one of the commonest causes of morbidity in persons with spinal cord injury after they have recovered from the initial effects of trauma. A systematic review of risk factors for urinary tract infection in adults with spinal cord dysfunction showed that persons using intermittent catheterisation had fewer infections than those with indwelling catheters did [1]. A follow-up of 128 patients with spinal cord injury for a period of 38 months showed the number of episodes of urinary infection per 100 patients per day was 2.72 for males with indwelling catheters, and 0.41 for males who performed clean intermittent catheterisation [2]. Although intermittent catheterisation may be associated with fewer episodes of urinary infection, some patients may develop urethral stricture as a result of frequent urethral trauma [3]. A review of 21 patients who had been performing clean intermittent catheterisation for over five years (mean length of use: 9.5 years) showed a rate of urethral stricture of $19 \%$, and of epididymitis of $28.5 \%$ [4]. A comparison of long-term renal function after spinal cord injury in 1114 persons who were injured between 1969 and 1994 showed that renal function was adequately preserved in the great majority of patients and did not appear to be influenced to any great extent by method of bladder management [5]. Therefore, it may seem reasonable when Yavuzer and associates [6] stated that the bladder management of spinal cord injury patients should be selected so as to be suitable to the patient's life style. Besides reducing morbidity, the method of bladder management should enhance the quality of life.

We observed complications with intermittent catheterisation, some of which may be unusual in clinical practice [7]. Hair introduced during catheterisation may act as a nidus for stone formation in the urinary bladder [8]. We studied urethral cytology of 50 patients of neurogenic vesical dysfunction who were practising clean, intermittent catheterisation. The changes observed included increased numbers of squamous epithelial cells, irregularity of their cell margins, neutrophil stickiness to the cell margins, cytoplasmic vacuolation, nuclear pyknosis and karyorrhexis. Increased numbers of inflammatory cells and the presence of bacterial flora were also noted [9].

The degree of urethral inflammation was compared between patients practising intermittent catheterisation and those using long-term indwelling urethral catheters. Urethral cytology was taken and the smear was stained by Papanicolaou's method. The number of neutrophil polymorphs and epithelial cells in three random high power fields in the urethral smear was counted and the percentage ratio of polymorphs to epithelial cells was calculated. 17 patients were performing intermittent catheterisation with a single use LoFric catheter. Urethral cytology revealed a percentage (mean ratio) ratio of polymorphs to epithelial cells of 0.04. In contrast, the ratio of polymorphs to epithelial cell was 159.3 in 11 patients with long-term urethral catheter. This study showed that use of a LoFric catheter for intermittent catheterisation was associated with significantly lesser degree of urethral inflammatory response when compared to the use of an indwelling urethral catheter [10]

We describe below a protocol of a prospective, cohort study to find out the incidence of urinary infection, predisposing factors for the occurrence of symptomatic urinary infection, and adverse events related to urinary catheter and urinary drainage system in male spinal cord injury patients using different methods of bladder drainage.

\section{Patients}

Regional Spinal Injuries Centre, Southport is a purposebuilt spinal unit situated in the Northwest coast of England. Spinal cord injury patients from north Wales, Merseyside, the Northwest of England, and Cumbria are referred to this unit for initial treatment. Thereafter, the spinal unit provides life-long follow-up for these patients. Patients are reviewed every 12 to 18 months, or earlier, if required. 1967 patients were registered with the Regional Spinal Injuries Centre, Southport up to 31 December 1997.

A preliminary letter about this study along with a self-addressed stamped envelope will be sent to all patients, who attended the spinal unit clinics during the past four years or, those who were discharged from the spinal unit during the last four years. (See additional file 1: 19 November 2001 Preliminary letter to Patient Urinary Infection Project.doc). A copy of Patient information sheet will be sent to those patients who evince interest in this study. (See additional file 2: 19 November 2001 Patient Information Sheet.doc). Those patients, who express their willingness to participate in this study, will come to the spinal unit for detailed discussion of the study with one of the investigators. If a patient agrees to participate in the study, informed consent will be taken from him and a letter will be sent the patient's general practitioner. (See additional file 3: 19 November 2001 Letter to General Practitioner Urine Infection Project.doc and additional file 4: 18 July 2001 Consent Symptomatic Urinary infection in SCI.doc)

Male spinal cord injury patients will be enrolled in this study in a consecutive manner until the target is reached. Following four groups of male patients will be recruited for this study. Table 1 
Table I:

\begin{tabular}{ll}
\hline Group 1 & Patients with indwelling urethral catheter \\
Group 2 & Patients performing intermittent catheterisation without wearing penile sheath \\
Group 3 & Patients performing intermittent catheterisation and wearing penile sheath \\
Group 4 & Patients with penile sheath drainage
\end{tabular}

The criteria for inclusion in this study are:

1. Male patients with neuropathic bladder due to spinal cord injury who are registered with the Regional Spinal Injuries Centre, Southport

\section{Age: 18 years or above}

3. Patients should be able to give informed consent for participation in the study. If a patient is unable to give written consent because of physical disability, a written affirmation of consent will be taken in his presence from his relative or carer.

4. Patients who are willing to be followed-up for a period of 36 months from the date of recruitment. This will involve fortnightly telephonic contact with the spinal unit and attendance in the spinal unit whenever they develop symptomatic urinary infection.

5. Patients should be willing to maintain a record of adverse events related to urinary catheter and the drainage system, and predisposing factors for the occurrence of urinary infection.

6. Patients, who are stabilised in a particular method of bladder drainage, and therefore, unlikely to make a permanent change in the method of bladder drainage (e.g. from penile sheath drainage to the use of long-term indwelling catheter) during a foreseeable future.

\section{Methods}

Data will be collected from each patient for a period of 36 months regarding the occurrence of symptomatic urinary infection, predisposing factors for the development of symptomatic urinary infection, and adverse events related to urinary catheter and urinary drainage system including the actual procedure of urethral catheterisation.

On enrolment, the participants will be given lists of (1) symptoms, which may be suggestive of acute urinary infection, (2) possible predisposing factors for the occurrence of urinary infection, and (3) adverse events related to urinary catheter and urine drainage system. The participants will be asked to record in a diary the occurrence of any of the above feature on a daily basis. An example is given in Table 2 .

A staff of the spinal injuries unit will contact the participants by telephone every two weeks on a mutually agreed day and time. The staff member will enquire the following:

$>$ Whether the participant noticed any adverse event related to urinary catheter and urine drainage system. The staff member will read out the list of adverse events one by one, and obtain a yes or no response for each item. The participant will be advised to refer to the diary for entries made during the preceding two weeks.

$>$ Whether the spinal cord injury patient developed any symptom suggestive of urinary infection. The staff member will read out the list of features to suggest symptomatic infection, and obtain a yes or no response from the participant for each item in the list. The participant will be advised to refer to the diary for entries made during the preceding two weeks.

$>$ Whether the participant noticed the presence or absence of predisposing factors for the development of urinary infection. The staff member will read out the list of predisposing factors, and get a yes or no response from the participant for each item. The participant will be advised to refer to the diary for entries made during the preceding two weeks.

A database will be created to enter the information obtained during this standardised interview over the telephone. The facts obtained during the telephonic interview will be entered in this database then and there. Confidential patient data will be stored in a relational database on computers with no external network access. Regular backup of the data onto external media will be required. The design and implementation of the database will allow the direct interactive capture of data from telephone interviews, with the data input screens providing the questions for the interviewer. Data will be coded to allow missing values to be identified. Data for analysis will be extracted by Structured Query Language (SQL) and will be transferred to specialist statistical software. In the four groups of patients identified in this study it is 
Table 2: An example of a daily record in the diary, which will be maintained by participants

Date: 12 October 2001

\section{Clinical features of urinary infection in $\mathrm{SCl}$ patients:}

Predisposing factors for the occurrence of urinary infection: Adverse events related to urinary catheter and drainage system:
Yes / No If yes, please specify the feature (see Table 2 for the complete list)

Yes I No If yes, please specify (see Table 3 for the complete list) Yes I No If yes, please specify (see Table 4 for a full list of adverse events)

Table 3: Criteria for symptomatic urinary infection Participants may have symptomatic urinary infection if a participant has recently developed one or more of the following symptoms:

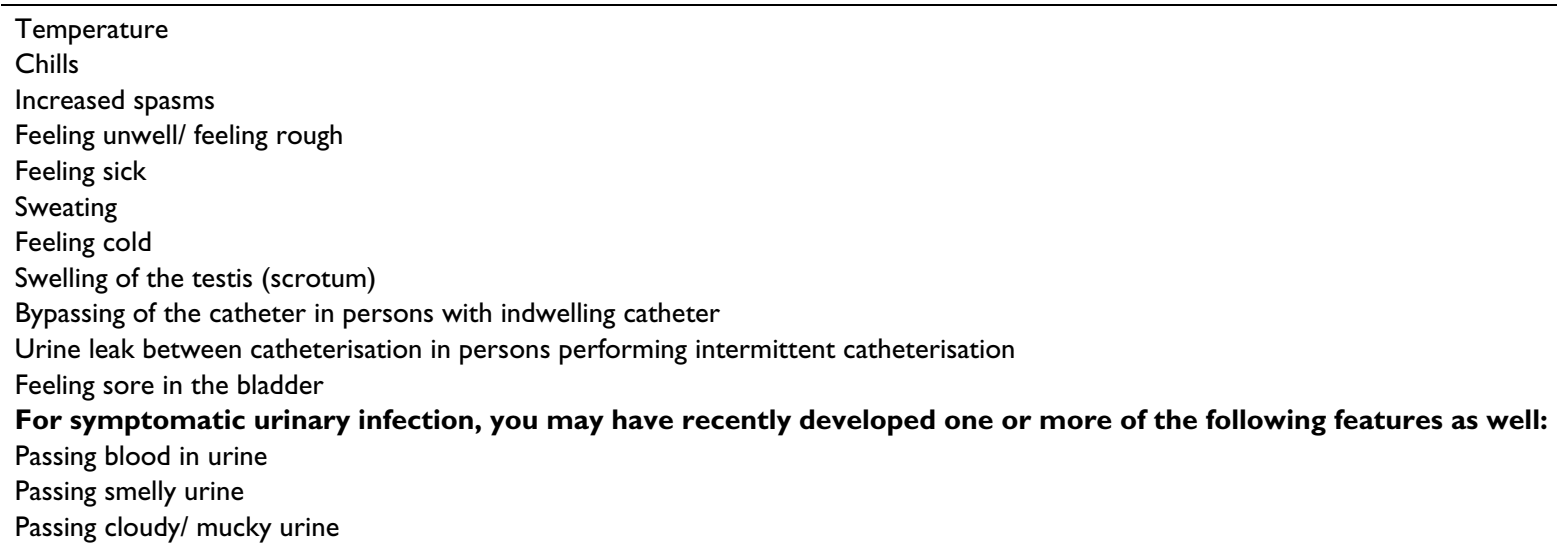

Table 4: Possible predisposing factors for the occurrence of Urinary Tract Infection in patients with Spinal Cord Injury

\begin{tabular}{lc}
\hline Change of indwelling urethral catheter & Yes / No \\
Improperly inserted urethral catheter & Yes / No \\
Catheter blockage or partial blockage of catheter & Yes / No \\
Flushing of catheter to relieve obstruction & Yes / No \\
Bladder stone & Yes / No \\
Kidney stone & Yes / No \\
Ureteric stone & Yes / No \\
Distension of urinary bladder e.g. drinking lot of fluids e.g. alcoholic beverages and failure to perform catheterisation (in those who & Yes / No \\
do intermittent catheterisation) & Yes / No \\
Failure to use a sterile catheter for intermittent catheterisation & Yes / No \\
Unhygienic toilet conditions for performing intermittent catheterisation & Yes / No \\
Unsatisfactory administration of bladder wash-outs & Yes / No \\
Decrease in fluid intake & Yes / No \\
Sexual activity which involved removal of urethral catheter prior to the sexual act, and insertion of a new catheter per urethra by & \\
the partner after completing sexual intercourse & Yes / No \\
Failure to change the leg bag as per schedule & Yes / No \\
Failure to change penile sheath daily &
\end{tabular}


Table 5: Adverse events pertaining to urinary catheter and urine drainage system in spinal cord injury patients

\begin{tabular}{lc}
\hline Bleeding from the urethra & Yes / No \\
Blood in the urine & Yes / No \\
Pull on the catheter & Yes / No \\
Partial slipping of an indwelling catheter & Yes / No \\
Spontaneous extrusion of an indwelling catheter & Yes / No \\
Forcible extrusion of an indwelling catheter & Yes / No \\
Catheter bypassing & Yes / No \\
Difficulty in inserting a new catheter per urethra & Yes / No \\
Split of the urethra (water-pipe) - traumatic hypospadias & Yes / No \\
Epididymitis/orchitis & Yes / No \\
Autonomic Dysreflexia & Yes / No \\
Predisposing factor for autonomic dysreflexia: Please specify: ---aing & \\
ache. Dysreflexia is often precipitated by obstruction to urine drainage. A SCl patient may develop dysreflexia when a catheter gets blocked or \\
when the urine bag becomes full and is not emptied promptly. The confirmation of the predisposing factor for the occurrence of dysreflexia is \\
prompt relief of dysreflexic symptoms when the predisposing factor is corrected e.g. urine bag is emptied, or a blocked catheter is changed. \\
Ulcer over the penis in those wearing a sheath & Yes / No \\
Skin excoriation over the penis in those wearing a sheath & Yes / No \\
Pressure mark on the thigh due to catheter or tubing & Yes / No \\
Stones in the urinary bladder & Yes / No \\
Obstruction to urinary drainage because of twisting of the penile sheath & Yes / No \\
Acute folding of the urethral catheter & Yes / No \\
Sharp bend of the drainage tube & Yes / No \\
Others (specify) & Yes / No
\end{tabular}

anticipated that the group with 'penile sheath drainage only' will have the least risk of infection and this group will be used as a control for the study. Logistic regression analysis is appropriate in sets of data where the dependent variable is dichotomous (for example infection or not infection) Data will be analysed using logistic regression with urinary infection (true/false) as the dependent variable, allowing comparison of the three remaining treatment regimes with the control. The relative risk of infection with $95 \%$ confidence interval and significance will be calculated for the independent variables collected by interview. Analysis of time dependent factors that influence the risk of infection will be performed using time series methods.

If a patient develops symptom(s) suggestive of urinary infection, he will be advised to come to the spinal unit for assessment and treatment, provided his condition permits travel to the spinal unit. Otherwise, he will go to the nearest accident \& emergency unit. When a participant attends the spinal unit with symptom(s) suggestive of urinary infection he will undergo urine and blood tests, and imaging studies of the urinary tract. All information pertaining to his attendance and subsequent admission in the spinal unit, if it becomes necessary, will be entered in the database, which is created specially for this research project.
Symptoms and signs, which may suggest that a spinal cord injury patient has developed acute urinary infection, are listed in Table 3. Possible predisposing factors for the occurrence of urinary infection in spinal cord injury patients are listed in Table 4. Adverse events, which are related to urinary catheter and urinary drainage system, are listed in Table 5 . The participants will be advised to make entries in the diary on a daily basis whether they developed a predisposing factor for the occurrence of urinary infection or any adverse event related to urinary catheter or urine drainage system. During telephonic contact every two weeks, the participants will be asked to refer to the diary when replying to the questions asked by the staff member. If a participant develops acute urinary infection, the staff member will find out whether the participant noticed any of the predisposing factors prior to the onset of symptomatic urinary infection.

When any participant develops urinary infection, he will be advised to come to the spinal unit, if the clinical condition permits. Blood and urine tests will be performed to confirm the diagnosis of acute urinary infection. Patients with acute urinary infection will be prescribed antibiotics as per the current clinical practice. We emphasise that no new treatment is being investigated in this study. All participants will continue to receive appropriate investigations and treatment for acute urinary infection as per the currently accepted clinical practice. 
The patients, their carers, partners, relatives, and the district nurses will be asked to contact the investigator if any of them feels that a participant (spinal cord injury patient) has developed urinary infection or any adverse event related to urinary drainage system. Patients who are suspected to have developed urinary infection and who are not very ill, will be advised to attend the spinal unit between 0900 and 1700 hours Monday to Friday as an out-patient after contacting one of the investigators over the phone. If a patient is not seriously ill and is able to come to the spinal unit, this patient will be examined by one of the doctors associated with this study on arrival at the spinal unit. A sample of urine will be sent for microbiology. A blood sample will be taken for full blood count and C-reactive protein to assess the degree of infection. The doctor may give a prescription for a course of antibiotics depending upon the clinical scenario. After the patient has gone home, a doctor or a nurse will speak to the patient over the phone on the next day and subsequently to inform him/her the results of urine and blood tests, and to ensure that the patient is making satisfactory recovery.

If a patient is ill and requires admission to a hospital, he/ she will be admitted to the spinal unit if a bed is available. When a patient or the carer contacts the spinal unit to say that the patient is seriously ill with suspected urinary infection, they will be advised to contact your GP on call or go to the nearest $\mathrm{A} \& \mathrm{E}$ as in the normal course of events. A General Practitioner may decide to send the patient who is seriously ill, to a local hospital. A doctor or a nurse from the spinal unit, who is involved in this study, will contact the local hospital, where the patient has been admitted. If a bed is available in the spinal unit, you will then be transferred to the spinal unit in Southport.

When a patient with suspected urinary infection attends the clinic in the spinal unit, he will be assessed in the following manner.

1. Clinical examination of the patient will be performed by one of the investigators. Each patient's symptoms and signs will be recorded by ticking the corresponding symptom/ sign in Table 2.

\section{A sample of urine will be sent for microbiology.}

3. A blood sample will be taken for measuring the C-reactive Protein level and full blood count.

4. Patient will be issued a prescription for antibiotic as per the decision of the attending physician.

5. Further investigations e.g. ultrasound scan of the urinary tract, X-ray of kidneys, ureter and bladder, intrave- nous urography, computerised tomography (CT) of abdomen will be carried out as per the current clinical practice.

The outcome of each episode of patient referral will be recorded.

- Did this patient have urinary infection or not (vide infra)?

-What was the urine microbiology report?

- What was the level of C-reactive protein?

- What was the white cell count?

- Did the clinician order additional tests

- X-ray of urinary tract to look for stones

- Ultrasound scan of the urinary tract

- Intravenous urography

- CT of the urinary tract

- Did this patient exhibit features autonomic dysreflexia during the acute phase of the illness?

- If a patient did not require hospitalisation, the duration of antibiotic therapy, and the duration of illness as perceived by the patient will be recorded.

- If a new diagnosis, e.g. presence of bladder stone was made after this episode of urinary infection, this will be recorded.

- If a patient is admitted to the spinal unit, the duration of hospital stay will be noted. Similarly, a record will be made regarding the antibiotic(s) prescribed, and any change in the management of urinary drainage will be noted.

- Changes bladder management will include

(1) Change from penile sheath drainage to indwelling urinary catheter drainage because the patient was retaining significant amount of urine in the bladder

(2) Increase in the frequency of intermittent catheterisations performed by the patient or the carer.

(3) Drugs prescribed to facilitate bladder emptying - e.g. alfuzosin 
- If a patient is admitted to another hospital, relevant information will be obtained from the treating hospital as soon as possible.

\section{Study Protocol}

Diagnosis of acute urinary infection

The gold standard for the diagnosis of acute urinary infection in the patients with spinal cord injury and neuropathic bladder are as follow: (1) positive urine microbiology and (2) presence of clinical features of urinary infection as listed in Table 3. Elevated levels of C-reactive protein and /or white cell count will provide further evidence for the presence of an acute infection.

Recording of predisposing factors for the occurrence of symptomatic urinary infection

The participant or his carers will record in a diary the occurrence of any predisposing factor for the development of urinary infection. Possible predisposing factors for the occurrence of urinary infection are listed in Table 4. The staff member, who contacts the participant once in two weeks, will specifically ask for the occurrence or non-occurrence of the predisposing factors during the preceding two weeks. The participants, their carers/partners, and the community nursing staff will be advised to inform the investigators in the spinal unit whenever a predisposing factor for the occurrence of urinary infection took place. The investigators will then speak to the participant and to the carers in order to obtain details of the predisposing factor. The information thus obtained will be entered in the database then and there.

\section{Recording of events related to urinary catheter}

The research subjects (patients with spinal cord injury) will be given a diary at the time of recruitment to the study. The investigators will discuss with the spinal cord injury patients participating in this study and their carers that they should make an entry daily whether any adverse event related to urinary catheter occurred or not. The patients or their carers will also note in the diary any change in the method of bladder drainage. For example, a patient who usually performs intermittent catheterisation may choose to put in an indwelling urethral catheter on Friday evening to enable him to go out during the weekend. He may remove the indwelling catheter on Monday and start performing intermittent catheterisation thereafter. This information will be documented in the diary by the participating subject or by the carer. If a participant is performing intermittent catheterisation, he will record any change in the number of catheterisations performed during a 24-hour period.

Recording of adverse events related to urinary drainage system The occurrence of any adverse event related to urinary catheters and urinary drainage system in spinal cord in-
Table 6: Number of patients who developed urethral split (traumatic hypospadias)

\begin{tabular}{ll}
\hline Patient Group & $\begin{array}{l}\text { Number of } \\
\text { patients }\end{array}$
\end{tabular}

Group I

Group 2

Group 3

Group 4

jury patients will be documented in a diary by the patient or by the carer. Possible adverse events related urinary catheters and urinary drainage system are listed in Table 5. Participants, their carers/partners, and community nursing staff will be advised to contact one of the investigators in the spinal unit as and when an adverse event occurs. The investigators will then speak to the participant and to the carers in order to obtain details of the adverse event. The staff member who rings the participant every two weeks will specifically ask for the presence or absence of each of the adverse event. If an adverse event had occurred during the past two weeks, this will be entered in the database.

\section{Communication with research subjects}

A staff member of the spinal unit will contact the participants over the phone once every two weeks on a day and time convenient to the participant which will be decided at the time of enrolment e.g. alternate Mondays at 1500 hours. The participants, his carers/partner, and the community nursing staff will be advised to contact the spinal unit whenever a patient develops symptoms or signs to suggest urinary infection, adverse event related to urinary catheter and urinary drainage system, or the predisposing factors for the development of urinary infection.

\section{Time-scale}

Recruitment of 200 persons with spinal cord injury will take place during the first two months. During the next 36 months, occurrence of symptomatic urinary infection, predisposing factors for the development of symptomatic urinary infection, and occurrence of adverse events related to urinary catheter, urinary drainage system, or to the technique of bladder catheterisation, will be observed and documented in the database. Data analysis and dissemination of the research findings will be performed during the subsequent two months. 
What is the primary end point?

Data will be collected from each patient for a period of 36 months from the date of recruitment to the study regarding:

$>$ Occurrence of symptomatic urinary infection

Predisposing factors for the development of symptomatic urinary infection

$>$ Adverse events related to the urinary catheter, urinary drainage system, and the actual procedure of urethral catheterisation

Interim analysis of the data will be carried out at the end of 6, 12 and 24 months. The number of episodes of infection per patient per month will be calculated for each group. The number of patient-episodes for each item in the three lists (features of symptomatic urinary infection, predisposing factors for the occurrence of urinary infection, and adverse events related to urinary catheter and urinary drainage system) will be calculated for the four groups. An example is given in Table 5 .

The number of episodes of admission due to (1) symptomatic urinary infection and, (2) adverse events related to urinary catheter and urinary drainage system, e.g. profuse bleeding due to urethral trauma during catheterisation, will be calculated for patients using different methods of bladder drainage. Complications of urinary infection, e.g. epididymo-orchitis, testicular abscess, hydronephrosis, pyonephrosis, perinephric abscess, will be recorded for each group of patients practising different methods of bladder drainage. Final analysis will be performed at the end of 36 months of observation.

\section{Conclusion}

We present the protocol of a prospective, cohort study in male spinal cord injury patients. Four groups of patients using (1) long-term indwelling urethral catheter, (2) penile sheath drainage, (3) intermittent catheterisation, and (4) a combination of penile sheath drainage and intermittent catheterisation will be recruited. This study is likely to provide valuable information regarding the occurrence of symptomatic urinary infection, predisposing factors for development of urinary infection, adverse events related to urinary catheter and urinary drainage system in the patients using different methods of bladder drainage.

\section{Sponsors of the research protocol}

We are grateful to the following sponsors who have kindly agreed to support this prospective, cohort study. Our sincere thanks to the sponsors whose names are given below in alphabetical order:

- AstraTech (financial support)

- B. Braun (financial support)

- Coloplast (financial support)

- DxU Limited (loan of ultrasound bladder scanner)

- Paraplegic Sports and Social Club (financial support)

- Renal Care \& Research Association (financial support)

- Sanofi-Synthelabo (financial support)

- Spinal Unit Action Group (financial support)

- SSL International (financial support)

- Tyco Healthcare (financial support)

\section{Competing interest}

One of the authors (BMS) received travel grants from AstraTech to attend international conferences. None of the authors received reimbursements, fees, or salary from an organisation that may in any way gain or lose financially from the publication of this paper in the past five years. None of the authors hold any stocks or shares in an organisation that may in any way gain or lose financially from the publication of this protocol.

\section{Additional material}

\section{Preliminary letter to patients}

Preliminary letter about this research project to be sent to male spinal cord injury patients

Click here for file

[http://www.biomedcentral.com/content/supplementary/I47I-2490-I2-SI.doc]

\section{Patient Information Sheet}

Letter to General Practitioner of spinal cord injury patient who will be participating in this study

Click here for file

[http://www.biomedcentral.com/content/supplementary/ |47I-2490-I2-S2.doc]

\section{Letter to General Practitioner}

Letter to General Practitioner of spinal cord injury patient who will be participating in this study

Click here for file

[http://www.biomedcentral.com/content/supplementary/ I47I-2490-I2-S3.doc] 


\section{Consent form}

Informed consent to be taken from participants before they are enrolled in the study

Click here for file

[http://www.biomedcentral.com/content/supplementary//47|-2490-I 2-S4.doc]

\section{Acknowledgement}

We profoundly thank Mr WS El Masry, Consultant in Spinal Injuries and Medical Director, Midlands Centre for Spinal Injuries, Oswestry, Shropshire; Mr John Reynard, Consultant Urologist, Oxford Radcliffe Hospital, The Churchill, Headington, Oxford; and Mr David Grundy, Consultant in Spinal Injuries, Salisbury, Wiltshire, England. We are grateful to Mr El Masry, Mr John Reynard and Mr David Grundy for reviewing this protocol and providing valuable comments.

We thank the reviewers of BioMed Central for their very useful advice, which enabled us to revise this manuscript accordingly.

\section{References}

I. Shekelle PG, Morton SC, dark KA, Pathak M, Vickrey BG: Systematic review of risk factors for urinary tract infection in adults with spinal cord dysfunction. J Spinal Cord Med 1999, 22:258-272

2. Esclarin De Ruz A, Garcia Leoni E, Herruzo Cabrera R: Epidemiology and risk factors for urinary tract infection in patients with spinal cord injury. J Urol 2000, 164:1285-1289

3. Mandal AK, Vaidyanathan S: Management of urethral stricture in patients practising clean intermittent catheterization. Int Urol Nephrol 1993, 25:395-399

4. Perrouin-Verbe B, Labat JJ, Richard I, Mauduyt de la Greve I, Buzelin JM, Mathe JF: Clean intermittent catheterisation from the acute period in spinal cord injury patients. Long term evaluation of urethral and genital tolerance. Paraplegia 1995, 33:619624

5. Sekar P, Wallace DD, Waites KB, DeVivo MJ, Lloyd LK, Stover SL, Dubovsky EV: Comparison of long-term renal function after spinal cord injury using different urinary management methods. Arch Phys Med Rehabil 1997, 78:992-997

6. Yavuzer G, Gok H, Tuncer S, Soygur T, Arikan N, Arasil T: Compliance with bladder management in spinal cord injury patients. Spinal Cord 2000, 38:762-765

7. Vaidyanathan S, Krishnan KR, Soni BM, Fraser MH: Unusual complications of intermittent self-catheterisation in spinal cord injury patients. Spinal Cord 1996, 34:745-747

8. Vaidyanathan S, Singh G, Sett P, Soni BM: Bladder stones of unusual shape in a male with paraplegia due to spinal cord injury who has been performing self-catheterisation. Spinal Cord 1999, 37:375-376

9. Nijhawan R, Vaidyanathan S, Radhika S, Dey P: Urethral cytology of patients practising clean, intermittent catheterisation for neurogenic vesical dysfunction. Indian-Journal-of-Urology 1994, II:13-19

10. Vaidyanathan S, Soni BM, Dundas S, Krishnan KR: Urethral cytology in spinal cord injury patients performing intermittent catheterisation. Paraplegia 1994, 32:493-500

Publish with BioMed Central and every scientist can read your work free of charge

"BioMedcentral will be the most significant development for disseminating the results of biomedical research in our lifetime."

Paul Nurse, Director-General, Imperial Cancer Research Fund

Publish with BMC and your research papers will be:

- available free of charge to the entire biomedical community

- peer reviewed and published immediately upon acceptance

- cited in PubMed and archived on PubMed Central

- yours - you keep the copyright

Submit your manuscript here:

http://www.biomedcentral.com/manuscript/
BioMedcentral.com editorial@biomedcentral.com 